\title{
PAPER
}

\section{Investigating the type and strength of emotion with music: An fMRI study}

\author{
Teruhisa Okuya ${ }^{1,5, *}$, Toshihiko Date ${ }^{2}$, Miwa Fukino ${ }^{3}$, Mikio Iwakawa ${ }^{1}$, \\ Kohji Sasabe ${ }^{1}$, Kumi Nagao ${ }^{4}$, Yuki Moriizumi ${ }^{5}$, \\ Iwaki Akiyama ${ }^{5}$ and Yoshiaki Watanabe ${ }^{5}$ \\ ${ }^{1}$ Eco Solutions Company, Engineering Division, Panasonic Corporation, \\ 1048 Kadoma, Kadoma, 571-8686 Japan \\ ${ }^{2} A V C$ Networks Company, Organization Staff R\&D Division, Panasonic Corporation, \\ 1-15 Matsuo-cho, Kadoma, 571-8504 Japan \\ ${ }^{3}$ Advanced Research Division, Panasonic Corporation, \\ 1006 Kadoma, Kadoma, 571-8501 Japan \\ ${ }^{4}$ Doshisha University, Life and Medical Sciences, \\ 1-3 Tatara Miyakodani, Kyotanabe, 610-0394 Japan \\ ${ }^{5}$ Doshisha University, Graduate School of Science and Engineering, \\ 1-3 Tatara Miyakodani, Kyotanabe, 610-0394 Japan
}

(Received 12 April 2016, Accepted for publication 13 November 2016)

\begin{abstract}
In this paper, we used blood oxygenation level-dependent (BOLD) signals and emotion ratings to investigate the relationship between the type and strength of emotion induced by musical stimuli. Our goal was to establish a quantitative emotional evaluation method that uses brain activity. In Experiment 1, 26 participants rated 60 pieces of music using a semantic differential scale and 20 pieces were chosen on the basis of Russell's circumplex model. In Experiment 2, we investigated the relationships between the type and strength of emotion and brain activity by asking 20 participants to listen to the pieces of music in a Magnetic Resonance Imaging (MRI) scanner. We identified brain regions for which the BOLD signal intensity was correlated with the ratings of emotions. As a result, the "Happy" rating was mainly correlated with activity in the superior temporal gyrus. The "Sad" rating was correlated with activity in the left thalamus. The "Fear" rating was mainly correlated with activity in the parahippocampal gyrus, insular cortex, and right amygdala. By focusing on activity in these brain regions, it may be possible to quantify the type and strength of emotions evoked by music.
\end{abstract}

Keywords: Emotion, Russell's circumplex model, Brain activity, fMRI, Pleasure, Arousal

PACS number: 43.64.+r [doi:10.1250/ast.38.120]

\section{INTRODUCTION}

A number of psychological and physiological measures have been proposed for assessing human emotions. For psychological measurements, questionnaires are frequently employed. However, questionnaires are disadvantageous in that they are often qualitative, require lengthy periods of scoring, and are subject to constraints regarding the interpretation of language. To address this, we used brain activity to investigate a quantitative emotional evaluation method.

Several studies have attempted to quantify individual emotions using brain activity, with techniques such as

*e-mail: okuya.teruhisa@jp.panasonic.com electroencephalography (EEG) and functional nearinfrared spectroscopy (fNIRS). In studies based on EEG, Musha et al. used the emotion spectrum analysis method [1] and Sato and Nakagawa used the emotion fractal dimension analysis method [2]. In the sensibility spectrum analysis technique, $\theta, \alpha$, and $\beta$ wave bands are used to estimate the degree of four basic emotions (anger, sadness, joy, relaxation) via the cross correlation coefficient between the electrodes. In the emotion fractal dimension analysis method, which is considered to be more accurate [2], emotions are assessed by applying the fractal dimension estimation method to brain wave difference signals. These techniques are based on EEG, which measures activity near the brain surface. However, brain regions related to emotions, such as the amygdala and hippocampus, are 


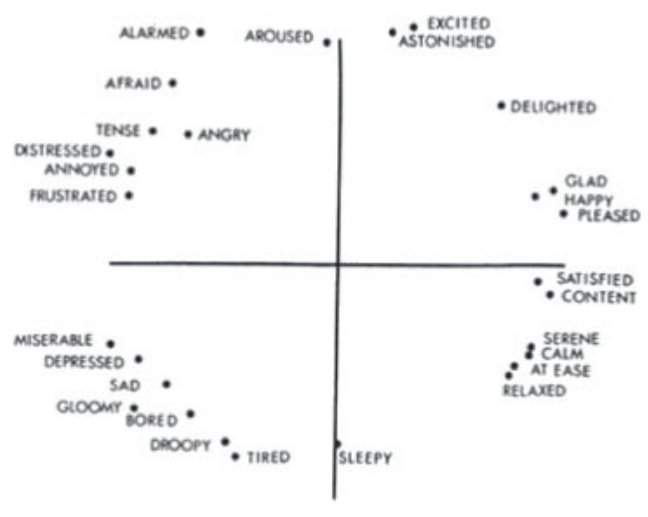

Fig. 1 Russell's circumplex model ( $x$-axis: pleasure, $y$-axis: arousal).

located deep within the brain [3]. Thus, it may be difficult to accurately evaluate emotions from EEG signals.

Indeed, functional magnetic resonance imaging (fMRI) may be more effective for evaluating deep brain activity. Many fMRI studies have addressed the neurophysiological mechanisms of emotions. For instance, happy music activates the superior temporal gyrus more strongly than neutral music $[4,5]$. Also, the hippocampus and parahippocampal gyrus have been reportedly activated by fearprovoking music [6-11]. However, many of the abovementioned studies focused only on the type of emotion, and only a few studies have evaluated emotion strength. For example, Trost et al. [12] focused on the intensity of emotions to explore a wider spectrum of affective responses reported during music listening. However, this experiment only explored the brain regions associated with the average intensity of over 31 people, and individual differences were not considered. To thoroughly assess a quantitative emotion evaluation method, it is necessary to focus on both the type and strength of individual's emotions. Therefore, in this study, we focused on brain regions associated with the type and strength of emotions elicited by music stimuli.

We conducted two experiments. In Experiment 1, we selected pieces of music to be used in an fMRI experiment by administering a questionnaire with a semantic differential (SD) scale. We performed principal component analysis and mapped each piece of music to Russell's circumplex model (Fig. 1) [13,14] to determine the type of emotion the piece evoked. Russell's circumplex model comprises two axes corresponding to pleasure and arousal. And attempts to cover the complete range of human emotions. In Experiment 2, participants listened to the pieces of music in an fMRI scanner. We then extracted the brain regions associated with both the type and the strength of emotions by comparing the emotional ratings and blood oxygenation level-dependent (BOLD) signals.

\section{EXPERIMENT 1 MUSIC SELECTION EXPERIMENT}

\subsection{Materials and Methods}

Professional sound engineers chose 50 pieces of music. The selections were intended to cover all areas included in Russell's circumplex model. Then, three experimenters performed preliminary experiments and added 10 pieces of music. Twenty six participants (22 men and four women, age 24-57, mean age 39.7, standard deviation 10.1) evaluated the 60 pieces of music individually using headphones. Each musical piece was about $30 \mathrm{~s}$ long and included instrumentation without vocals. The participants listened to the music and then rated the pieces via a questionnaire. Participants were instructed to evaluate their emotions absolutely after listening. The questionnaire, which had a seven-level SD scale, was written in Japanese on response sheets generated on the basis of previous reports [15]. The questionnaire included 19 adjective pairs such as "Merry-Solemn," "Happy-Sad," "Calm-Fear." Participants were allowed to adjust the music volume to a comfortable level. Participants were prohibited from rating the music while listening and were asked to rest as necessary. The pieces of music were presented in a random order that was different for each subject.

\subsection{Results}

We performed principal component analysis using a correlation matrix. Table 1 shows the results of the factor loadings. The contribution rate of the first and second components was $64 \%$, and the contribution rate was $71 \%$ when the third component was included. The first principal component included adjectives such as "Merry," "Lively," and "Up tempo," which accounted for pleasure and arousal. The second component included adjectives such as "Fear," "Tension," and "Anxiety," which accounted for displeasure and arousal. The third main component included "Fresh," which was not related to pleasure or arousal. We constructed Russell's circumplex model using the first and second principal components, which were associated with pleasure and arousal. Specifically, we placed the first principal component on the $y$-axis and the second principal component on the negative $x$-axis. We then rotated the function by $26.1^{\circ}$ clockwise to move the adjective "Pleasure" as close as possible to the $x$-axis and the adjective "Aroused" as close as possible to the $y$-axis [16]. This process served to associate the $x$-axis with 'Pleasantness' and the $y$-axis with 'arousal.' Figure 2 shows the emotional space created by the rotation. The adjective "Happy" is in the first quadrant, and "Calm" is located in the fourth quadrant, as in Russell's circumplex model (shown in Fig. 1). The other adjectives are located similarly to in Russell's circumplex model. Therefore, we 
Table 1 Semantic differential (SD) scales and factor loadings.

\begin{tabular}{|c|c|c|c|c|}
\hline \multicolumn{2}{|r|}{ SD scale } & \multicolumn{3}{|c|}{ Factor } \\
\hline English & Japanease & Component 1 & mponen & mponent 3 \\
\hline Merry-Solemn & うきうきしたーしんみりした & 0.86 & -0.15 & -0.13 \\
\hline Lively-Quiet & にぎやかなー静かな & 0.86 & 0.16 & -0.13 \\
\hline Up tempo-Slow tempo & アップテンポの-スローテンポの & 0.85 & 0.16 & -0.10 \\
\hline Happy-Sad & 楽しい-悲しい & 0.78 & -0.42 & -0.01 \\
\hline Energetic-Peaceful & 勢いのある-落ち着いた & 0.77 & 0.41 & -0.11 \\
\hline Cheerful-Dismal & 陽気なー陰気な & 0.74 & -0.51 & -0.04 \\
\hline Bright-Dark & 明るい-暗い & 0.72 & -0.55 & -0.07 \\
\hline Comical-Tearful & 笑える-泣ける & 0.70 & -0.16 & -0.23 \\
\hline Aroused-Sleepy & 覚醒する-鎮静する & 0.68 & 0.45 & -0.12 \\
\hline Hot-Cool & 熱い-さめた & 0.65 & 0.05 & 0.28 \\
\hline Fierce-Gentle & 激しい-穏やかな & 0.64 & 0.57 & -0.04 \\
\hline Powerful-Weak & 力強い-弱弱しい & 0.54 & 0.35 & 0.36 \\
\hline Fear-Peace & 恐れ-安らぎ & -0.01 & 0.87 & -0.18 \\
\hline Tension-Relax & 緊張-リラックス & 0.11 & 0.87 & -0.12 \\
\hline Anxiety-Ease & 不安-安心 & -0.16 & 0.86 & -0.19 \\
\hline Unpleasure-Pleasure & 不快な-快適な & -0.26 & 0.76 & -0.32 \\
\hline Heavy-Light & 重量感のある-軽量感のある & -0.34 & 0.57 & 0.39 \\
\hline Exciting-Boring & 刺激的な-平凡な & 0.48 & 0.49 & 0.42 \\
\hline Fresh-Predictable & 新鮮なーありきたりな & 0.37 & 0.22 & 0.60 \\
\hline \multicolumn{2}{|c|}{ Contribution rate } & 0.38 & 0.64 & 0.71 \\
\hline
\end{tabular}

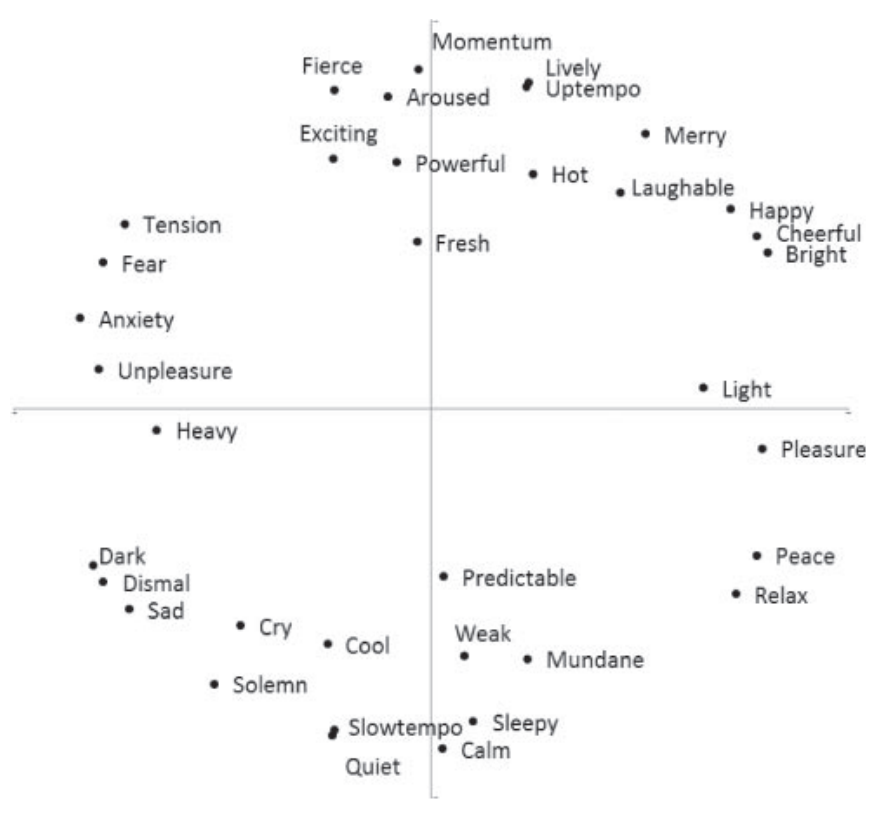

Fig. 2 Configured space.

confirmed that we were able to construct a space similar to that in Russell's circumplex model.

By placing the average scores for each piece of music into this space, we were able to consider the strength of the emotion that each piece evoked. Figure 3 shows the average values for the 60 pieces of music, located in the above-mentioned space. Happy music tended to be located in the first quadrant, fearful music was located in the second quadrant, sad music was located in the third

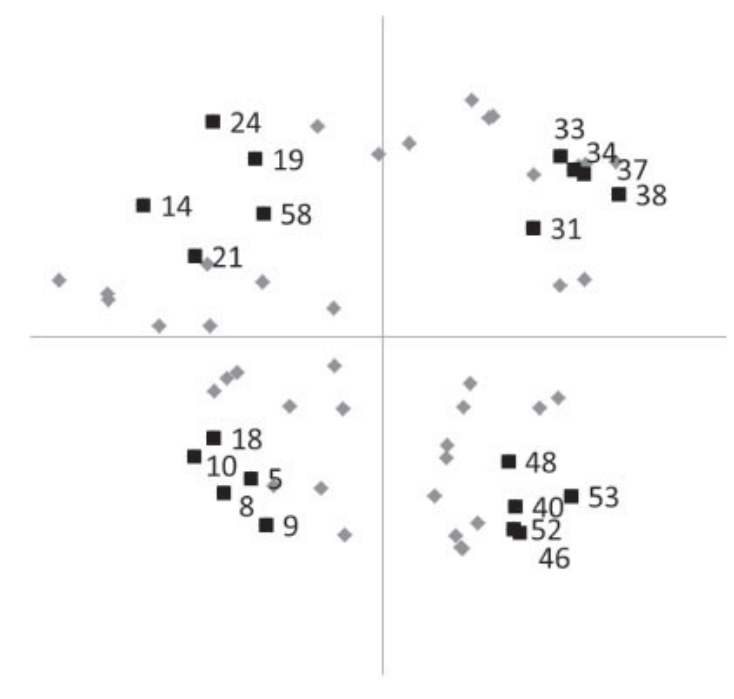

Fig. 3 Locations of all 60 pieces of music (black dots: 20 pieces chosen for MRI experiment). quadrant, and calm music was located in the fourth quadrant. The musical stimuli with a fast tempo were rated as having high arousal (happy or fearful music), and the musical stimuli with a slow tempo were rated as having low arousal (sad or calm music). Also, musical stimuli in a major scale were rated as having high pleasantness and musical stimuli in a minor scale were rated as having low pleasantness. Finally, we chose five pieces of music from each quadrant. We attempted to maximize the number of different instruments used, thus focusing on brain regions 
Table 2 Twenty pieces of music chosen for MRI experiment.

\begin{tabular}{|c|c|c|c|c|c|}
\hline Music No & Emotion Type & Music Title & Tempo & Tonality & Instrument \\
\hline 31 & Happy & All Wrapped Up & 128 & C Major & organ, sax, drum, bass \\
\hline 33 & Happy & SAMBA de Koisite & 118 & D Major & saxophone, drum, bass \\
\hline 34 & Happy & Garden Party & 120 & D Major & trumpet, drum, bass \\
\hline 37 & Happy & FESTA!! & 118 & D Major & guitar, percussion \\
\hline 38 & Happy & Tijuana Taxi & 102 & F Major & trumpet, drum, guitar \\
\hline 5 & Sad & Cancer & 87 & D minor & piano \\
\hline 8 & Sad & Tuioku & 51 & A minor & cello, strings \\
\hline 9 & Sad & Naval & 90 & D minor & piano \\
\hline 10 & Sad & Solitude & 85 & G minor & strings, bass \\
\hline 18 & Sad & Inside Elevation & 78 & D minor & accordion, synthesizer \\
\hline 40 & Calm & Captain's Café & 88 & C Major & ukulele, guitar, bass \\
\hline 46 & Calm & Emma & 67 & F Major & piano \\
\hline 48 & Calm & Soothing & 90 & F Major & synthesizer, guitar, bass \\
\hline 52 & Calm & Olema Waltz & 85 & D Major & piano, strings \\
\hline 53 & Calm & GendouNoSekai & 70 & C Major & harp, synthesizer, strings \\
\hline 14 & Fear & Half Day & 73 & Atonal & synthesizer \\
\hline 21 & Fear & Disc Wars & 82 & D minor & synthesizer, timpani \\
\hline 58 & Fear & Weird and Scary & 110 & A b minor & synthesizer \\
\hline 24 & Fear & False Awakening Suite & 133 & $\begin{array}{l}\text { D minor } \\
\rightarrow F \text { minor }\end{array}$ & bass \\
\hline 19 & Fear & High Volume & 171 & C minor & synthesizer, drum \\
\hline
\end{tabular}

related specifically to emotion, rather than make the brain process different types of musical sounds. Table 2 shows the 20 pieces of music that were chosen for Experiment 2.

\section{EXPERIMENT 2 MRI experiment}

\subsection{Materials and Methods}

A total of 20 pieces of music were chosen from Experiment 1 to be presented inside the MRI scanner. Participants listened to the music via MRI-compatible piezoelectric ceramic headphones (Kiyohara Optics Inc.). All 20 participants (18 males and two females, age 22-55, mean age 31.3, standard deviation 10.7), different from those in Experiment 1, were right-handed and had no history of hearing problems. The experiment had a block design, designed by Presentation software (Neurobehavioral Systems, Ltd.), which began with $30 \mathrm{~s}$ of rest, after which the subject listened to a piece of music (presented in random order) for $30 \mathrm{~s}$. The subjects then rated their emotions within $12 \mathrm{~s}$ using MRI-compatible buttons (Package 904: Current Designs, Inc.). A questionnaire was projected onto a screen using a projector. To reduce the mental burden on the participants, we presented only two adjective pairs ("Happy-Sad," "Calm-Fear," also used in Experiment 1). The participants rated their emotions absolutely using an 11-point visual analogue scale (VAS). Experiments were performed in two trials, and each subject evaluated a total of 20 pieces of music.

\section{2. fMRI Measurement Conditions}

fMRI measurements were conducted using a $1.5 \mathrm{~T}$ scanner (Echelon Vega: Hitachi Medical Corporation) at Doshisha University. Participants were placed on the scanner bed in a supine position. To prevent postural adjustments and to attenuate the noise and vibration of the scanner, foam cushions were placed around the arms of the participants. We acquired 27 oblique slices covering the whole brain (field of view $192 \mathrm{~mm}$; thickness $5 \mathrm{~mm}$ ) using a sequential gradient echo-planar imaging (EPI) sequence (TR 3,000 ms; flip angle $90^{\circ}$ ) sensitive to BOLD contrast. Before the fMRI measurement, participants were informed about the study protocol, signed a written consent form, filled in a safety questionnaire, and were required to remove any ferromagnetic material before entering the magnet bore. Participants were encouraged to relax in the magnet bore while concentrating on the music stimuli. The study procedures were approved by the ethical committee of Doshisha University (No. 1365) and complied with the Helsinki Declaration.

\subsection{Data Analysis}

We excluded data for two participants that exhibited a head movement of more than $2.5 \mathrm{~mm}$. Thus, we analyzed data for a total of 18 participants (17 males and one female, age 22-55, mean age 31.3, standard deviation 11.2). We used MATLAB 2012b (The MathWorks, Natick, MA) and SPM12 (Wellcome Department of Cognitive Neurology, London) software. Images for each subject were realigned, slice timing was corrected to the 14th slice, and the images were spatially normalized onto the Montreal Neurological Institute (MNI) template and spatially smoothed (Gaussian 

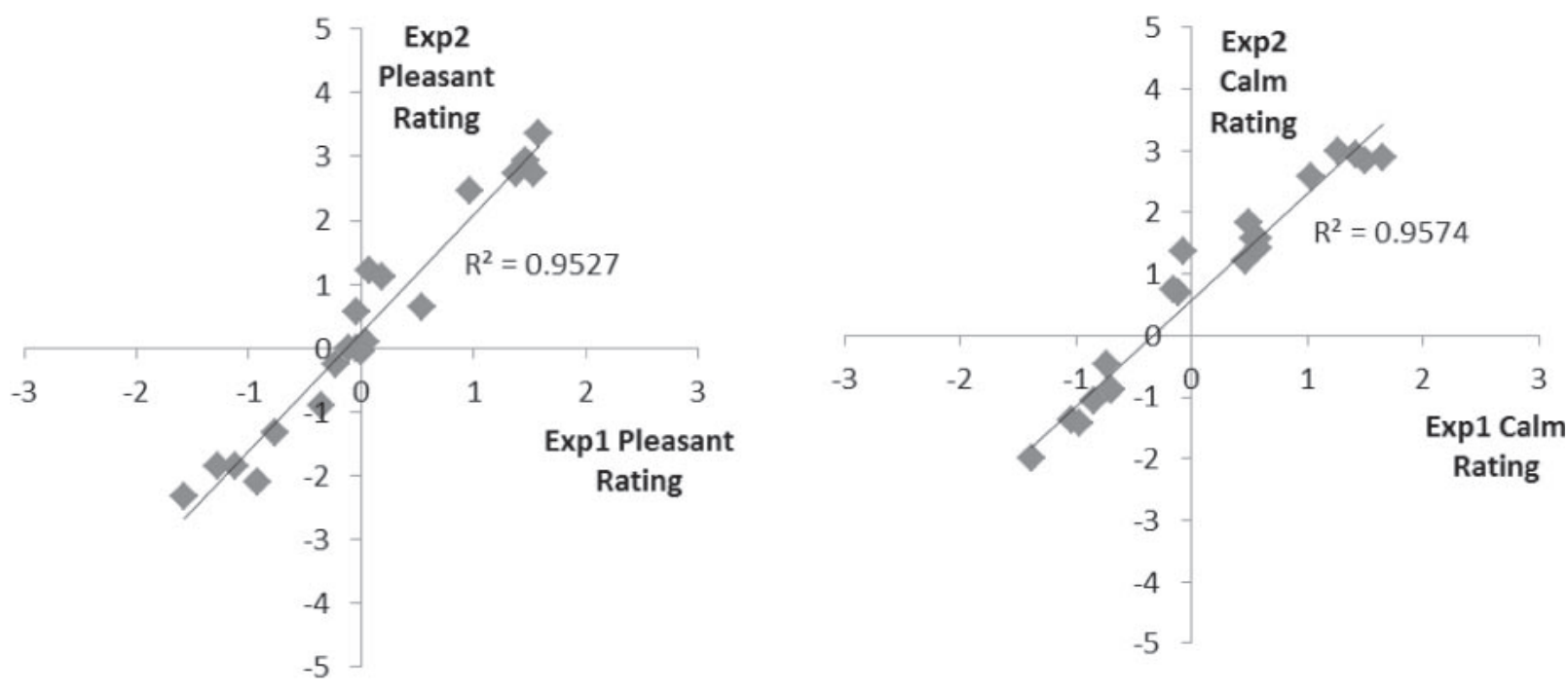

Fig. 4 Comparison of ratings from Experiments 1 and Experiments 2.

filter with an FWHM of $7 \mathrm{~mm}$ ). fMRI responses were modeled using a canonical hemodynamic response function (HRF) with time dispersion and temporally filtered using a high-pass filter of $128 \mathrm{~Hz}$ to minimize scanner drift. The six parameters for residual movement were modeled as regressors of no interest. Specifically, using parametric modulation, we analyzed the BOLD signals collected for all participants to determine the brain regions for which activity corresponded to the ratings of emotion obtained from the questionnaire $(p<0.001$, none).

\subsection{Results}

Figure 4 shows the correlation between the ratings of emotion for the 20 pieces of music in Experiments 1 and 2. The rating of "Happy-Sad" had $R^{2}=0.9527$ and the rating of "Calm-Fear" had $R^{2}=0.9574$. Thus, we were able to confirm that the stimuli elicited nearly identical emotions in the two experiments.

Table 3 shows the brain regions with a voxel size of $k \geqq 3$ for which BOLD signals were correlated between emotional ratings. Each brain region was labeled using automated anatomical labeling (AAL) [17]. As parts of the brain associated with the rating of "Happy," we identified the angular gyrus, calcarine sulcus, middle cingulate gyrus, posterior cingulate gyrus, precuneus, and superior temporal gyrus. The brain region associated with the rating of "Sad" was the left thalamus. As the parts of the brain associated with the rating of "Fear," we extracted the right amygdala, inferior frontal gyrus, middle frontal gyrus, insula, lingual gyrus, parahippocampal gyrus, precuneus, gyrus rectus, rolandic operculum, supplementary motor area, superior temporal gyrus, left thalamus, and vermis. No region was associated with the rating of “Calm" (Fig. 5).

\section{DISCUSSION}

In Experiment 1, we administered a questionnaire based on the SD scale and then conducted principal component analysis. As a result, the first principal component was associated with pleasure and arousal, and the second principal component was associated with displeasure and arousal. In many previous studies, pleasure and arousal were extracted as first or second principal components. However, in this experiment, they were mixed in the first and second components. This is likely because principal component analysis determines the largest dispersion of the data with respect to the first principal component. The axis for the second largest dispersion of the data is correlated with the second principal component and not with the first principal component. Because of the wide range of emotions elicited by the music chosen in this experiment, we found that both pleasure and arousal were widely dispersed. Accordingly, the mixed components were extracted as the first and second principal components. Therefore, we rotated the space formed by the first and second components to situate "Pleasure" near the $x$-axis and "Arousal" near the $y$-axis. As a result, we formed a space that mirrored Russell's circumplex model. By locating the evaluated music in this space, we were able to classify the 60 pieces of music more accurately than if we had done so according to the emotions that each piece evoked.

In Experiment 2, we used only two pairs of adjectives ("Happy-Sad," "Calm-Fear"). As both pairs of adjective ratings produced $R^{2}>0.95$ for the correlation between Experiment 1 and Experiment 2, we were able to verify that the emotions in Experiment 1 were the same as those elicited in Experiment 2. 
Table 3 Brain regions associated with emotional ratings $(p<0.001$, none).

\begin{tabular}{|c|c|c|c|c|c|c|c|}
\hline Emotion & Brain region & $\begin{array}{c}\text { MNI } \\
\text { coordinates } \\
x y z(\mathrm{~mm})\end{array}$ & $k$ & $\mathrm{BA}$ & $Z$ value & $T$ & $p$ (unc) \\
\hline \multirow[t]{7}{*}{ HAPPY } & Angular_L & {$\left[\begin{array}{lll}-42 & -58 & 24\end{array}\right]$} & 4 & 39 & 3.19 & 3.85 & 0.0007 \\
\hline & Calcarine_R & {$\left[\begin{array}{lll}2 & -64 & 16\end{array}\right]$} & 4 & 31 & 3.15 & 3.78 & 0.0008 \\
\hline & Cingulum_Mid_L & {$\left[\begin{array}{lll}-2 & -12 & 40\end{array}\right]$} & 3 & 24 & 3.23 & 3.92 & 0.0006 \\
\hline & Cingulum_Post_L & {$\left[\begin{array}{lll}-2 & -38 & 24\end{array}\right]$} & 3 & 23 & 3.13 & 3.74 & 0.0009 \\
\hline & Precuneus_L & {$\left[\begin{array}{lll}-12 & -40 & 2\end{array}\right]$} & 9 & & 3.36 & 4.13 & 0.0004 \\
\hline & Temporal_Sup_L & {$\left[\begin{array}{llll}-44 & -6 & -10\end{array}\right]$} & 6 & 21 & 3.37 & 4.15 & 0.0004 \\
\hline & Temporal_Sup_L & {$\left[\begin{array}{lll}-46 & -34 & 16\end{array}\right]$} & 11 & 41 & 3.28 & 4.00 & 0.0005 \\
\hline SAD & Thalamus_L & {$\left[\begin{array}{lll}-12 & -20 & 14\end{array}\right]$} & 7 & & 3.15 & 3.75 & 0.0008 \\
\hline \multirow[t]{19}{*}{ FEAR } & Amygdala_R & {$\left[\begin{array}{lll}24 & 2 & -16\end{array}\right]$} & 11 & 34 & 3.28 & 4.12 & 0.0005 \\
\hline & Insula_R & {$\left[\begin{array}{lll}34 & -8 & 14\end{array}\right]$} & 92 & & 4.07 & 5.80 & 0.0000 \\
\hline & Insula_R & {$\left[\begin{array}{lll}36 & 2 & 14\end{array}\right]$} & & 13 & 3.68 & 4.90 & 0.0001 \\
\hline & Rolandic_Oper_R & {$\left[\begin{array}{lll}44 & -6 & 12\end{array}\right]$} & & 13 & 3.31 & 4.18 & 0.0005 \\
\hline & Frontal_Inf_Tri_L & {$\left[\begin{array}{lll}-34 & 32 & 12\end{array}\right]$} & 5 & 46 & 3.32 & 4.20 & 0.0004 \\
\hline & Frontal_Mid_R & {$\left[\begin{array}{lll}40 & 26 & 20\end{array}\right]$} & 34 & 46 & 3.39 & 4.32 & 0.0004 \\
\hline & Frontal_Inf_Tri_R & {$\left[\begin{array}{lll}48 & 28 & 20\end{array}\right]$} & & 46 & 3.29 & 4.14 & 0.0005 \\
\hline & Frontal_Med_Orb_R & {$\left[\begin{array}{lll}6 & 54 & -8\end{array}\right]$} & 3 & 10 & 3.45 & 4.44 & 0.0003 \\
\hline & Frontal_Mid_R & {$\left[\begin{array}{lll}26 & 48 & 26\end{array}\right]$} & 11 & 10 & 3.64 & 4.82 & 0.0001 \\
\hline & Insula_L & {$\left[\begin{array}{lll}-36 & 10 & 6\end{array}\right]$} & 12 & 13 & 3.54 & 4.62 & 0.0002 \\
\hline & Lingual_R & {$[18-36-4]$} & 40 & 30 & 3.50 & 4.54 & 0.0002 \\
\hline & Parahippocampal_R & {$[20-14-22]$} & 22 & 28 & 3.32 & 4.19 & 0.0005 \\
\hline & Precuneus_L & {$\left[\begin{array}{lll}-8 & -52 & 18\end{array}\right]$} & 13 & 30 & 3.76 & 5.07 & 0.0001 \\
\hline & Rectus_L & {$\left[\begin{array}{lll}-18 & 12 & -16\end{array}\right]$} & 6 & 47 & 3.48 & 4.49 & 0.0003 \\
\hline & Rolandic_Oper_R & {$\left[\begin{array}{lll}50 & 6 & 14\end{array}\right]$} & 19 & 44 & 3.65 & 4.83 & 0.0001 \\
\hline & Supp_Motor_Area_R & {$\left[\begin{array}{lll}14 & -6 & 70\end{array}\right]$} & 3 & 6 & 3.16 & 3.90 & 0.0008 \\
\hline & Temporal_Sup_L & {$\left[\begin{array}{lll}-58 & -6 & 0\end{array}\right]$} & 4 & 22 & 3.19 & 3.95 & 0.0007 \\
\hline & Thalamus_L & {$\left[\begin{array}{lll}-14 & -30 & 8\end{array}\right]$} & 5 & & 3.28 & 4.11 & 0.0005 \\
\hline & Vermis_4_5 & {$\left[\begin{array}{lll}-2 & -46 & -4\end{array}\right]$} & 16 & & 3.34 & 4.23 & 0.0004 \\
\hline
\end{tabular}

CALM

We found that the activity in the superior temporal gyrus correlated with the rating of "Happy." This region has been implicated in sound perception and spokenlanguage processing. In addition, a significant increase in activity in this site is often reported during exposure to happy versus neutral music [4,5]. Thus, this region may be important for evaluation of the enjoyment of music.

We also found that the activity in the left thalamus correlated with the rating of "Sad." This site is known to be a relay point for sensory information and also controls arousal. Additionally, this region is more significantly

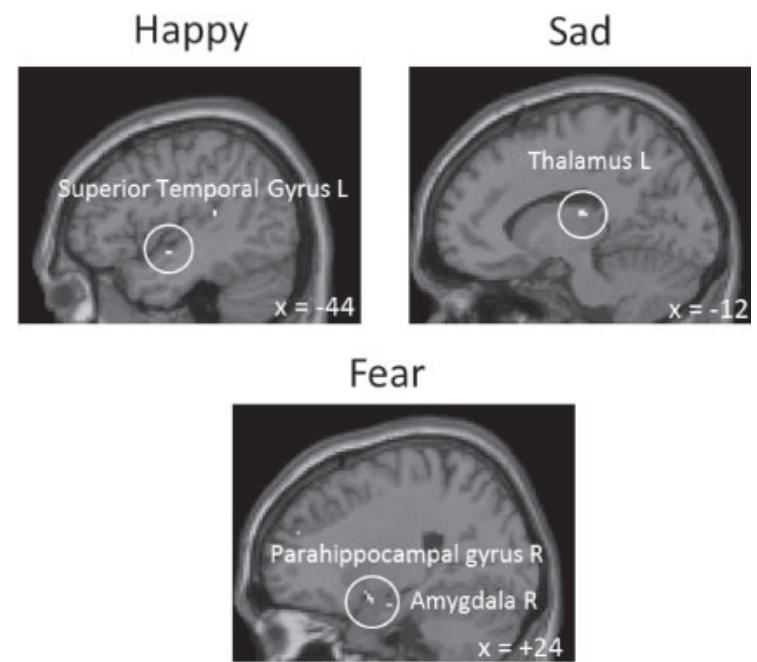

Fig. 5 Examples of brain regions associated with emotions $(p<0.001$, none).

activated in response to sad versus neutral music [5]. Interestingly, a large meta-analysis of 105 neuroimaging studies of facial expressions found that the left thalamus is one of the few structures (along with a region in the visual cortex and the left insula) that are consistently active when processing sad faces [18]. This consistency hints at the cross-modal nature of basic emotions evoked by visual or auditory stimuli [19].

We found that the activity in the insular cortex was associated with the rating of "Fear." This region is known for sensory processing, such as pain sensation and discomfort. Additionally, this region is involved in impulses, such as hunger and craving, and is activated by a combination of fearful images and music [20]. Furthermore, we found that the activity in the right amygdala was associated with fear. This region is known to be involved in emotional reactions and emotional memory. The right part of the amygdala has also been previously associated with fear [21]. We identified fear-related activation in the parahippocampal gyrus. This region is related to learning and memory [6-11] and shows diminished emotional sensitivity to dissonant music $[22,23]$. In addition, this region is activated when viewing unpleasant images [24]. Furthermore, this site is strongly coupled with the amygdala [25] and is relevant to emotional expression.

We found that no region whose voxel size was $k \geqq 3$ was associated with the evaluation of "Calm." However, the lower frontal lobe, which voxel size was $k=1$, was associated. This site is a part of the Broca's area, which is known to be involved in speech and grammar. In addition, this site is activated when listening to highly pleasurable music [26]. "Calm" is related to high ratings of pleasure and sleep in Russell's circumplex model. Thus, it is 
reasonable that the lower frontal lobe was identified as related to this dimension. However, since this region had a voxel size of $k=1$, we must consider that this result is not siginificant.

Many previous studies have reported on brain regions associated with emotion types. In these studies, brain regions are generally identified by comparing brain activity between two types of emotions or by comparing an emotion with a 'neutral' state, and differences in emotional ratings between individuals were not considered. However, in this experiment, we revealed that the regions listed above are related not only to the type of emotion but also to the strength of the emotion. By analyzing these brain regions, it may be possible to determine the type and strength of individual emotions evoked by music. The limitation of this study is that our findings are only applicable to the emotions evoked by auditory stimuli. Furthermore, it is necessary to confirm whether similar results can be obtained even when musical stimuli are played by speakers rather than headphones.

\section{CONCLUSION}

In Experiment 1, we rated the emotion evoked by 60 pieces of music using Russell's circumplex model. We then chose 20 pieces of music for our fMRI experiment (Experiment 2). We confirmed that the same emotions were evoked in Experiments 1 and 2. For the 20 pieces of music, we extracted the brain regions for which the type and strength of emotions were correlated. As a result, the "Happy" rating was mainly correlated with activity in the superior temporal gyrus, "Sad" with the left thalamus, and "Fear" with the right amygdala and parahippocampal gyrus.

The brain regions extracted in this experiment were associated with both the type and strength of emotions. Furthermore, these regions exhibited greater activation when the emotions were more strongly expressed. By analyzing activity in these brain regions, it may be possible to determine the type and strength of emotions evoked by music.

\section{REFERENCES}

[1] T. Musha, Y. Terasaki, H. A. Haque and G. A. Ivanitsky, "Feature extraction from EEGs associated with emotions," Artif. Life Robotics, 1, 15-19 (1997).

[2] T. Sato and M. Nakagawa, "Quantification of emotion using fractal dimension analysis," Tech. Rep. IECE HIP, P2002-45 (2002) (in Japanese).

[3] S. Koelsch, "Towards a neural basis of music-evoked emotions," Trends Cognit. Sci., 14, 131-137 (2010).

[4] M. T. Mitterschiffthaler, C. H. Fu, J. A. Dalton, C. M. Andrew and S. C. Williams, "A functional MRI study of happy and sad affective states induced by classical music," Hum. Brain Mapp., 28, 1150-1162 (2007).

[5] E. Brattico, V. Alluri, B. Bogert, T. Jacobsen, N. Vartiainen, S. Nieminen and M. Tervaniemi, "A functional MRI study of happy and sad emotions in music with and without lyrics," Front. Psychol., 2, 10.3389 (2011).

[6] S. Zola-Morgan, L. R. Squire, D. G. Amaral and W. A. Suzuki, "Lesions of perirhinal and parahippocampal cortex that spare the amygdala and hippocampal formation produce severe memory impairment," J. Neurosci., 9, 4355-4370 (1989).

[7] M. Bunsey and H. Eichenbaum, "Critical role of the parahippocampal region for paired-associate learning in rats," Behav. Neurosci., 107, 740-747 (1993).

[8] G. K. Aguirre, J. A. Detre, D. C. Alsop and M. D’Esposito, "The parahippocampus subserves topographical learning in man," Cereb. Cortex, 6, 823-829 (1996).

[9] D. Sammler, M. Grigutsch, T. Fritz and S. Koelsch, "Music and emotion: Electrophysiological correlates of the processing of pleasant and unpleasant music," Psychophysiology, 44, 293-304 (2007).

[10] T. Ball, B. Rahm, S. B. Eickhoff, A. Schulze-Bonhage, O. Speck and I. Mutschler, "Response properties of human amygdala subregions: Evidence based on functional MRI combined with probabilistic anatomical maps," PLoS One, 3, 307 (2007)

[11] A. J. Blood, R. J. Zatorre, P. Bermudez and A. C. Evans, "Emotional responses to pleasant and unpleasant music correlate with activity in paralimbic brain regions," Nat. Neurosci., 2, 382-387 (1999).

[12] W. Trost, T. Ethofer, M. Zentner and P. Vuilleumier, "Mapping aesthetic musical emotions in the brain," Cereb Cortex, 22, 2769-2783 (2012).

[13] J. A. Russell, "A circumplex model of affect," J. Pers. Soc. Psychol., 39, 1161-1178 (1980).

[14] J. A. Russell, A. Weiss and G. A. Mendelsohn, "Affect grid: A single item scale of pleasure and arousal," J. Pers. Soc. Psychol., 57, 493-502 (1989).

[15] T. Sugihara, K. Morimoto, T. Kawamura, M. Shimada and T. Kurokawa, "Selecting Kansei words for use in a Kansei-based music retrieval system for young people," J. Jpn. Soc. Kansei Eng., 5, 127-134 (2005) (in Japanese).

[16] H. Abdi and L. J. Williams, "Principal component analysis," WIREs Comput. Stat., 2, 433-459 (2010).

[17] N. Tzourio-Mazoyer, B. Landeau, D. Papathanassiou, F. Crivello, O. Etard, N. Delcroix, B. Mazoyer and M. Joliot, "Automated anatomical labeling of activations in SPM using a macroscopic anatomical parcellation of the MNI MRI singlesubject brain," NeuroImage., 15, 273-289 (2002).

[18] P. Fusar-Poli, A. Placentino, F. Carletti, P. Landi, P. Allen, S. Surguladze, F. Benedetti, M. Abbamonte, R. Gasparotti, F. Barale, J. Perez, P. McGuire and P. Politi, "Functional atlas of emotional faces processing: A voxel-based meta-analysis of 105 functional magnetic resonance imaging studies," $J$. Psychiatry Neurosci., 34, 418-432 (2009).

[19] S. Koelsch, "Towards a neural basis of music-evoked emotions," Trends Cognit. Sci., 14, 131-137 (2010).

[20] T. Baumgartner, K. Lutz, C. F. Schmidt and L. Jancke, "The emotional power of music: How music enhances the feeling of affective pictures," Brain Res., 1075, 151-164 (2006).

[21] K. B. Baker and J. J. Kim, "Amygdalar lateralization in fear conditioning: Evidence for greater involvement of the right amygdala," Behav. Neurosci., 118, 15-23 (2004).

[22] N. Gosselin, S. Samson, R. Adolphs, M. Noulhiane, M. Roy, D. Hasboun, M. Baulac and I. Peretz, "Emotional responses to unpleasant music correlates with damage to the parahippocampal cortex," Brain, 129, 2585-2592 (2006).

[23] S. Khalfa, M. Guye, I. Peretz, F. Chapon, N. Girard, P. Chauvel and C. Liegeois-Chauvel, "Evidence of lateralized anteromedial temporal structures involvement in musical 
emotion processing," Neuropsychologia, 46, 2485-2493 (2008).

[24] R. D. Lane, E. M. Reiman, M. M. Bradley, P. J. Lang, G. L. Ahern, R. J. Davidson and G. E. Schwartz, "Neuroanatomical correlates of pleasant and unpleasant emotion," Neuropsychologia, 35, 1437-1444 (1997).
[25] L. Stefanacci, W. A. Suzuki and D. G. Amaral, "Organization of connections between the amygdaloid complex and the perirhinal and parahippocampal cortices in macaque monkeys," J. Comp. Neurol., 375, 552-582 (1996).

[26] S. Koelsch, "Investigating emotion with music: An fMRI study," Hum. Brain Mapp., 27, 239-250 (2006). 